\title{
Saúde materno-infantil em comunidades quilombolas no norte de Minas Gerais
}

\author{
Maternal infant health in quilombo communities \\ in northern Minas Gerais, Brazil
}

\author{
Stéphany Ketlin Mendes Oliveira ${ }^{1}$, Mayane Moura Pereira ${ }^{1}$, \\ Daniel Antunes Freitas², Antônio Prates Caldeira ${ }^{3}$
}

\begin{abstract}
Resumo
Objetivou-se descrever indicadores de saúde materno-infantil em comunidades quilombolas ao norte de Minas Gerais. Trata-se de estudo transversal, de base populacional. Os dados foram coletados nos domicílios, alocados aleatoriamente, incluindo 411 mulheres com idade entre 18 e 49 anos e 234 crianças com idade igual ou inferior a cinco anos. A maioria das famílias $(73,7 \%)$ referiu uma renda mensal inferior a um salário mínimo, e quase metade das mulheres entrevistadas $(49,2 \%)$ tinha escolaridade inferior a quatro anos. O acesso à água tratada, esgotamento sanitário e coleta de lixo era limitado. A maioria das mulheres $(52,1 \%)$ relatou a primeira gestação na adolescência e 35\% relataram quatro ou mais gestações. A realização de exames preventivos para o câncer de colo uterino se mostrou irregular e 15,1\% das mulheres nunca haviam feito o exame. Em relação ao ciclo gravídico-puerperal, 23,5\% das mulheres referiram menos de seis consultas de pré-natal, 37,2\% relataram início do pré-natal após o primeiro trimestre de gestação e 44,4\% não fizeram consulta puerperal. Em relação às crianças, $15 \%$ nasceram com baixo peso, 9,4\% tinham problemas crônicos de saúde e 40,6\% não faziam uso regular de vitamina A. Os resultados destacam condições críticas de acesso aos cuidados de saúde.
\end{abstract}

Palavras-chave: saúde materno-infantil; comunidades vulneráveis; grupo com ancestrais do continente africano; desigualdades em saúde.

\begin{abstract}
We aimed to describe indicators of maternal and child health in this communities in northern Minas Gerais, Brazil. This is crosssectional, population-based study. Data were collected in households randomly sampled including 411 women aged 18 to 49 years and 234 children aged less than five years. Most families (73.7\%) reported a monthly income less than minimum wage and nearly half of respondents (49.2\%) had less than four years of schooling. Accesses to drinking water, sewage and garbage collection were limited. Most women (52.1\%) reported the first pregnancy as adolescents and $35 \%$ reported four or more pregnancies. Preventive examinations for cervical cancer showed irregular and $15.1 \%$ of women had never been screened. In relation to pregnancy and childbirth, $23.5 \%$ of women reported fewer than six prenatal consultations, $37.2 \%$ reported onset of prenatal care after the first trimester and $44.4 \%$ did attended a puerperal consultation. With regard to children, $15 \%$ had low birth weight, $9.4 \%$ had chronic health problems and $40.6 \%$ were taking no vitamin A. The results highlight critical conditions access to public services, including access to care health.
\end{abstract}

Keywords: maternal and child health; vulnerable groups; African continental ancestry group; health inequalities.

Trabalho realizado na região Norte de Minas Gerais, Universidade Estadual de Montes Claros (UNIMONTES) - Montes Claros (MG), Brasil. ${ }^{1}$ Mestranda do Programa de Pós-graduação em Ciências da Saúde, UNIMONTES; Professora das Faculdades Unidas do Norte de Minas (FUNORTE) - Montes Claros (MG), Brasil.

${ }^{2}$ Doutorando do Programa de Pós-graduação em Ciências da Saúde, UNIMONTES; Professor da FUNORTE - Montes Claros (MG), Brasil.

${ }^{3}$ Doutor em Ciências da Saúde pela Universidade Federal de Minas Gerais (UFMG); Professor do Programa de Pós-graduação em Ciências da Saúde,

UNIMONTES - Montes Claros (MG), Brasil.

Endereço para correspondência: Antônio Prates Caldeira - Avenida Cula Mangabeira, 562 - Santo Expedito - CEP: 39401-001 - Montes Claros (MG), Brasil E-mail: antonio.caldeira@unimontes.br

Fonte de financiamento: Fundação de Amparo à Pesquisa do Estado de Minas Gerais (FAPEMIG) - Processo: CDS - APQ - 00645-11.

Conflito de interesses: nada a declarar. 


\section{INTRODUÇÃO}

Mulheres e crianças representam, quase sempre, a parcela mais vulnerável da sociedade diante de condições adversas de vida. Durante muitos anos, no Brasil, o modelo econômico adotado promoveu uma significativa concentração de renda, com reflexos bastante negativos sobre os indicadores de saúde materno-infantil ${ }^{1}$. Embora as condições atuais sejam mais adequadas e tenham sido registradas melhorias na qualidade da atenção ao binômio mãe-filho nos últimos anos, o acesso igualitário para uma adequada assistência à gestação e ao parto ainda se mostra insuficiente ${ }^{1,2}$.

Um aspecto que por muitos anos foi negligenciado pela literatura nacional, em relação às iniquidades da atenção à saúde da mulher e da criança, é a influência da cor da pele. Durante muito tempo, acreditava-se que os estudos focados nos determinantes socioeconômicos poderiam destacar a influência do racismo sobre os indicadores de saúde, fato que não mais é aceitável ${ }^{3,4}$. Estudos nacionais mais recentes desconstruíram a ideia de uma "democracia racial", apontando diferenciais importantes entre brancos e negros, com indicadores de saúde inaceitáveis para a população negra ${ }^{5-7}$ e quilombola ${ }^{8,9}$.

No conjunto da população negra brasileira, é possível identificar que o grupo das comunidades quilombolas parece ainda mais negligenciado. Quase sempre localizadas em áreas rurais, essas comunidades, originalmente constituídas por descendentes de escravos, sobreviveram à margem dos benefícios sociais, preservando a dependência da terra para sua reprodução física, social, econômica e cultural ${ }^{10}$.

A escassa literatura, pelo menos na área da saúde, sobre as comunidades quilombolas destaca também a invisibilidade dessas comunidades aos olhos da academia. Existem poucos estudos que abordam a questão da saúde materno-infantil para comunidades negras ou, mais especificamente, para comunidades quilombolas. Alguns estudos limitam-se a comunidades isoladas e não permitem a generalização de dados. Em Minas Gerais, a distribuição da população quilombola é heterogênea, mas cerca de $40 \%$ das comunidades reconhecidas pela Fundação Palmares encontra-se localizada ao norte do Estado. Não existem estudos que tenham abordado tais comunidades de forma global, buscando identificar similaridades ou condições de risco para os agravos de saúde. O presente estudo objetivou descrever indicadores de saúde materno-infantis para uma amostra representativa de mulheres e crianças de comunidades quilombolas ao norte o Estado de Minas Gerais.

\section{MÉTODOS}

Trata-se de um estudo transversal, de base populacional, descritivo e de abordagem quantitativa. A área de abrangência foi a macrorregião norte de Minas, que incorpora 86 municípios, dos quais 20 possuem comunidades quilombolas. Inicialmente, efetuou-se o levantamento de todas as comunidades quilombolas reconhecidas pela Fundação Palmares existentes na região norte de Minas Gerais. A partir das 33 comunidades identificadas, elaborou-se um plano amostral para alocação de residências que fossem representativas de todas as comunidades.

Nesse sentido, a unidade amostral do presente estudo foi a residência. $\mathrm{O}$ cálculo amostral considerou uma prevalência de $50 \%$ para os eventos estudados, pois se trata de um valor conservador, que fornece o maior número amostral. A margem de erro aceitável foi de 5\%, e o nível de confiança de $95 \%$. Para a população estimada nas comunidades quilombolas da região, o cálculo amostral definiu a necessidade de 378 domicílios a serem visitados. Como o processo de alocação da amostra foi por conglomerados (comunidades), o número de domicílios foi multiplicado por 1,5 (fator de correção do desenho) e acrescido de $15 \%$ para eventuais perdas (domicílios sem moradores ou recusas). Definiu-se, assim, a necessidade de visitas a 652 domicílios.

Os domicílios foram selecionados por amostragem probabilística em dois estágios. Em um primeiro momento, as comunidades foram alocadas de forma aleatória. Em cada comunidade, selecionou-se um ponto de referência, a partir do qual os domicílios foram visitados, construindo-se uma espiral imaginária, dada a variabilidade da ocupação do espaço, pois as comunidades estavam quase sempre localizadas em zonas rurais. O número de residências selecionadas em cada localidade foi proporcional ao tamanho dela.

Em cada residência, identificou-se, quando presente, uma moradora com idade igual ou superior a 18 anos e inferior a 50 anos, e uma criança com idade igual ou inferior a 5 anos, para coleta de dados relativos aos objetivos do presente estudo. Outros moradores do domicílio também foram alocados para pesquisa, mas não fazem parte do presente estudo.

Foram visitados 756 domicílios em 33 comunidades. Nesses domicílios foram identificadas por sorteio 411 mulheres com idade entre 18 e 49 anos e 234 crianças com idade igual ou inferior a 5 anos.

O projeto de investigação foi avaliado e aprovado pelo Comitê de Ética em Pesquisa da Universidade Estadual de Montes Claros. Todos os respondentes consentiram com a participação no estudo, registrando sua concordância no termo de consentimento livre e esclarecido com assinatura ou com digital, quando não sabiam escrever. $\mathrm{O}$ acesso às comunidades foi mediado pelo representante estadual, fato que possibilitou participação universal, sem recusas. 
Todas as entrevistas foram conduzidas por profissionais da área da saúde nas próprias residências dos respondentes. Foram utilizados instrumentos de coleta de dados já validados, baseados em pesquisas similares. Os dados foram avaliados a partir de sua apresentação em tabelas, com números absolutos e proporcionais.

\section{RESULTADOS}

A Tabela 1 caracteriza as famílias estudadas, com base no acesso a bens de consumo duráveis e serviços públicos. Entre os bens de consumo duráveis no domicílio, foram mais comuns o registro de televisão, geladeira, rádio e telefone celular. Entre os serviços públicos, exceto pelo maior acesso à energia elétrica, registrou-se limitado acesso para as famílias quilombolas.

Tabela 1. Acessibilidade a bens de consumo duráveis e serviços públicos entre famílias quilombolas no Norte de Minas Gerais, 2013

\begin{tabular}{|c|c|c|}
\hline Variáveis & $\mathbf{n}$ & $\%$ \\
\hline \multicolumn{3}{|c|}{ Bens de consumo duráveis no domicílio* } \\
\hline Rádio & 294 & 71,5 \\
\hline Geladeira & 365 & 88,8 \\
\hline Máquina de lavar & 47 & 11,4 \\
\hline Televisão & 370 & 90,0 \\
\hline Telefone fixo & 21 & 5,1 \\
\hline Telefone celular & 250 & 60,8 \\
\hline Computador & 16 & 3,9 \\
\hline Motocicleta & 88 & 21,4 \\
\hline Carro & 18 & 4,4 \\
\hline \multicolumn{3}{|l|}{ Destino do lixo } \\
\hline Queimado & 231 & 56,2 \\
\hline Coletado em caçamba & 105 & 25,5 \\
\hline Coletado pelo serviço público & 20 & 4,9 \\
\hline Enterrado & 17 & 4,1 \\
\hline Jogado em terreno baldio & 38 & 9,2 \\
\hline \multicolumn{3}{|l|}{ Destino do esgoto } \\
\hline Fossa séptica & 304 & 74,0 \\
\hline Fossa rudimentar & 59 & 14,4 \\
\hline Vala & 22 & 5,4 \\
\hline Rio ou lago/outros & 17 & 4,1 \\
\hline \multicolumn{3}{|l|}{ Energia elétrica } \\
\hline Com medidor exclusivo & 365 & 88,8 \\
\hline Com medidor compartilhado & 31 & 7,5 \\
\hline Sem medidor & 9 & 2,2 \\
\hline Sem acesso & 6 & 1,5 \\
\hline \multicolumn{3}{|l|}{ Abastecimento de água } \\
\hline Poço ou nascente & 289 & 70,3 \\
\hline Rede geral & 57 & 13,9 \\
\hline Carro pipa & 39 & 9,5 \\
\hline Rios, açudes, lagos & 26 & 6,3 \\
\hline
\end{tabular}

${ }^{*} \mathrm{O}$ percentual de cada bem/produto se refere ao total de famílias entrevistadas.

A caracterização sociodemográfica das mulheres participantes do estudo é apresentada na Tabela 2. A maior parte das entrevistadas referia renda familiar inferior a um salário mínimo, era casada ou vivia em união estável e declarou escolaridade inferior a oito anos.

A Tabela 3 descreve as principais variáveis associadas à saúde reprodutiva das mulheres quilombolas avaliadas. Observou-se que $214(52,1 \%)$ mulheres relataram a primeira gestação com idade inferior a 20 anos e 144 (35,0\%) declararam já ter vivenciado 4 ou mais gestações. Registrou-se ainda que $48(11,7 \%)$ mulheres entrevistadas nunca realizaram um exame preventivo para o câncer do colo uterino e 266 (64,7\%) informaram que nunca tiveram suas mamas examinadas por um profissional de saúde. Entre as entrevistadas, 109 (26,5\%) relataram histórico de contracepção permanente (laqueadura tubária ou histerectomia).

As Tabelas 4 e 5 descrevem as características da assistência ao ciclo gravídico-puerperal e das crianças quilombolas avaliadas. Essa avaliação apenas foi realizada para as mães de crianças menores de cinco anos. Entre os aspectos mais importantes, destaca-se que 55 (23,5\%) mães fizeram menos de 6 consultas de atendimento pré-natal e, entre as que tiveram atendimento pré-natal, 77 (32,9\%) iniciaram esse atendimento após o terceiro mês de gestação. Entre as crianças, 35 (15,0\%) nasceram com peso abaixo de $2.500 \mathrm{~g}$. Os principais problemas crônicos apresentados pelas crianças foram: asma/bronquite, anemia falciforme e problemas ortopédicos.

Tabela 2. Características demográficas e sociais de mulheres quilombolas no norte de Minas Gerais, 2013

\begin{tabular}{lcc} 
Variáveis & $\mathbf{n}$ & $\%$ \\
Idade & & \\
18-29 anos & 136 & 33,1 \\
30-39 anos & 149 & 36,2 \\
40-49 anos & 126 & 30,7 \\
Cor da pele & & \\
$\quad$ Preta & 343 & 83,5 \\
Parda & 56 & 13,6 \\
$\quad$ Amarela/branca/indígena & 12 & 2,9 \\
Situação conjugal & & \\
Solteira/separada/viúva & 126 & 30,7 \\
Casada ou união estável & 285 & 69,3 \\
Renda familiar & & \\
$\quad<1$ SM & 306 & 73,7 \\
1-1,9 SM & 97 & 22,9 \\
$\quad \geq 2$ SM & 8 & 1,9 \\
Escolaridade & & \\
Nenhuma & & \\
1-4 anos & 41 & 10,0 \\
5-8 anos & 161 & 39,2 \\
$\geq 9$ anos & 85 & 20,7 \\
\hline SM: salário minimo & 124 & 30,2 \\
\hline
\end{tabular}


Tabela 3. Variáveis associadas à saúde reprodutiva de mulheres quilombolas no norte de Minas Gerais, 2013

\begin{tabular}{|c|c|c|}
\hline Variáveis & $\mathbf{n}$ & $\%$ \\
\hline \multicolumn{3}{|l|}{ Idade da $1^{\text {a }}$ gestação } \\
\hline$<20$ anos & 214 & 52,1 \\
\hline 20-24 anos & 113 & 27,5 \\
\hline $25-29$ anos & 29 & 7,1 \\
\hline$\geq 30$ anos & 7 & 1,7 \\
\hline Nunca esteve grávida & 48 & 11,7 \\
\hline \multicolumn{3}{|l|}{ Número de gestações } \\
\hline Nenhuma & 48 & 11,7 \\
\hline 1 & 77 & 18,7 \\
\hline 2 & 74 & 18,0 \\
\hline 3 & 68 & 16,5 \\
\hline 4 ou mais & 144 & 35,0 \\
\hline \multicolumn{3}{|l|}{ Histórico de aborto } \\
\hline Sim & 82 & 20,0 \\
\hline Não & 281 & 68,4 \\
\hline Não se aplica & 48 & 11,7 \\
\hline \multicolumn{3}{|l|}{ Realização prévia de PCCU* } \\
\hline Sim & 349 & 84,9 \\
\hline Não & 62 & 15,1 \\
\hline \multicolumn{3}{|l|}{ Frequência dos exames preventivos } \\
\hline Anualmente ou menos & 257 & 62,5 \\
\hline Uma vez a cada 2 anos & 56 & 13,6 \\
\hline Uma vez a cada 3 anos ou mais & 36 & 8,8 \\
\hline Nunca fez & 62 & 15,1 \\
\hline \multicolumn{3}{|l|}{ Uso de contraceptivos } \\
\hline Pílulas & 120 & 29,2 \\
\hline Outros & 44 & 10,7 \\
\hline Nenhum & 247 & 60,1 \\
\hline \multicolumn{3}{|l|}{ Exame clínico das mamas } \\
\hline Sim & 145 & 35,3 \\
\hline Não & 266 & 64,7 \\
\hline \multicolumn{3}{|c|}{ Intervenção cirúrgica gineco-obstétrica prévia ${ }^{* *}$} \\
\hline Cesárea & 85 & 20,7 \\
\hline Laqueadura tubária & 100 & 24,3 \\
\hline Histerectomia & 9 & 2,2 \\
\hline Outros & 7 & 1,7 \\
\hline Nenhuma & 260 & 63,3 \\
\hline
\end{tabular}

*Exame preventivo de câncer de colo uterino.

${ }^{* *}$ Percentual $>100 \%$ : algumas mulheres tiveram mais de um procedimento.

\section{DISCUSSÃO}

As comunidades quilombolas se caracterizam por particularidades étnicas que as distinguem do restante da sociedade. Historicamente, esse grupo esteve à margem dos benefícios sociais governamentais por muitos anos. Seu reconhecimento oficial, com a outorga de direitos plenos de cidadania somente ocorreu a partir da Constituição Federal de 1988, e políticas especiais de assistência social e saúde para esse grupo só foram implantadas mais tardiamente ${ }^{11}$. Os resultados do presente estudo destacam que esse grupo continua marcado por processos de discriminação e exclusão, e que muitos dos indicadores
Tabela 4. Características da atenção ao ciclo gravídico-puerperal para mulheres quilombolas no norte de Minas Gerais, $2013(n=234)$

\section{Variáveis}

Consultas de pré-natal

$\begin{array}{lcc}<4 & 20 & 8,5 \\ 4 \text { a } 5 & 35 & 15,0 \\ 6 \text { ou mais } & 179 & 76,5 \\ \text { ocal do pré-natal } & & \\ \text { PSF } & 170 & 72,6 \\ \text { Centro de Saúde } & 42 & 17,9 \\ \text { Hospital } & 19 & 8,1 \\ \text { Não fez pré-natal } & 3 & 1,3\end{array}$

Quem acompanhou o pré-natal

$\begin{array}{lll}\text { Médico generalista ou médico do PSF } & 166 \quad 70,9\end{array}$

Enfermeiro do PSF $59 \quad 25,2$

$\begin{array}{lll}\text { Ginecologista } & 6 & 2,6\end{array}$

Não fez pré-natal $\quad 3 \quad 1,3$

Início do pré-natal

$\leq 3$ meses $\quad 154 \quad 65,8$

$\begin{array}{lll}>3 \text { meses } & 77 & 32,9\end{array}$

Não fez $\quad 3 \quad 1,3$

Uso de vitaminas na gestação

Não $\quad 21 \quad 9,0$

Sim $213 \quad 91,0$

Tipo de parto

$\begin{array}{lll}\text { Normal } & 185 & 79,1\end{array}$

$\begin{array}{lll}\text { Cesariano } & 49 & 20,9\end{array}$

Consulta de puerpério

$\begin{array}{lll}\text { Não } & 104 & 44,4 \\ \text { Sim } & 130 & 55,6\end{array}$

Idade da primeira consulta puericultura $\begin{array}{lll}\leq 15 \text { dias } & 60 & 25,6\end{array}$

15-30 dias $\quad 125 \quad 53,4$

$\begin{array}{lll}>30 \text { dias } & 49 & 20,9\end{array}$

PSF: Programa de Saúde da Família.

de saúde materno-infantil ainda estão distantes dos valores eticamente aceitáveis.

Registrou-se uma acentuada diferença entre o acesso aos bens de consumo duráveis no domicílio e o acesso aos serviços públicos. Enquanto o primeiro parece ter sido ampliado nos últimos anos, o segundo mantêm-se com indicadores críticos. O restrito acesso à água tratada e a um adequado esgotamento sanitário satisfatório imprime, sobretudo às crianças das comunidades, um maior risco de contrair doenças decorrentes da agressão ao meio ambiente ${ }^{12}$.

As comunidades avaliadas revelaram-se sem adequado atendimento em relação aos serviços de saneamento básico, coleta de lixo e acesso à água tratada. Naturalmente, as condições geográficas das comunidades quilombolas, quase sempre localizadas em áreas rurais, representam uma grande dificuldade, mas o histórico de segregação dessas comunidades pode estar contribuindo para a perpetuação das condições observadas. Situação similar de inexistência de condições sanitárias 
Tabela 5. Características das crianças quilombolas no norte de Minas Gerais, 2013

\begin{tabular}{|c|c|c|}
\hline Variáveis & $\mathbf{n}$ & $\%$ \\
\hline \multicolumn{3}{|l|}{ Idade da criança } \\
\hline$<12$ meses & 42 & 17,9 \\
\hline $12-23$ meses & 48 & 20,5 \\
\hline 24-35 meses & 56 & 23,9 \\
\hline $36-47$ meses & 51 & 21,8 \\
\hline $48-59$ meses & 37 & 15,8 \\
\hline \multicolumn{3}{|l|}{ Cor da pele } \\
\hline Preta & 202 & 86,3 \\
\hline Parda & 23 & 9,8 \\
\hline Amarela/branca/indígena & 9 & 3,9 \\
\hline \multicolumn{3}{|l|}{ Peso de nascimento } \\
\hline$<2.499 \mathrm{~g}$ & 35 & 15,0 \\
\hline $2.500-2.999 \mathrm{~g}$ & 66 & 28,2 \\
\hline $3.000-3.999 \mathrm{~g}$ & 115 & 49,1 \\
\hline$\geq 4.000 \mathrm{~g}$ & 18 & 7,7 \\
\hline \multicolumn{3}{|l|}{ Número pessoas na casa } \\
\hline$<4$ pessoas & 48 & 20,5 \\
\hline 4 a 7 pessoas & 154 & 65,8 \\
\hline$\geq 8$ & 32 & 13,7 \\
\hline \multicolumn{3}{|l|}{ Presença do pai } \\
\hline Sim & 185 & 79,1 \\
\hline Não & 49 & 20,9 \\
\hline \multicolumn{3}{|c|}{$\begin{array}{l}\text { Registro de problema crônico de saúde da } \\
\text { criança }\end{array}$} \\
\hline Sim & 12 & 9,4 \\
\hline Não & 212 & 90,6 \\
\hline \multicolumn{3}{|l|}{ Uso regular de vitamina $\mathrm{A}$} \\
\hline Sim & 119 & 50,9 \\
\hline Não & 95 & 40,6 \\
\hline Não se aplica & 20 & 8,5 \\
\hline \multicolumn{3}{|l|}{$\begin{array}{l}\text { Benefício social do governo } \\
\text { ("bolsa família") }\end{array}$} \\
\hline Sim & 212 & 90,6 \\
\hline Não & 12 & 9,4 \\
\hline \multicolumn{3}{|l|}{ Vacinação em dia } \\
\hline Sim & 229 & 97,9 \\
\hline Não & 5 & 2,1 \\
\hline
\end{tabular}

apropriadas, como água corrente limpa para consumo humano e esgoto sanitário tratado, foi registrada na comunidade de Caiana dos Crioulos, na Paraíba ${ }^{13}$.

As características das mulheres quilombolas registram uma população jovem, cuja maioria encontra-se em união conjugal. Socialmente, existem indicadores que reforçam os aspectos de pobreza, pois cerca de 70\% dessas mulheres referiam uma renda familiar inferior a um salário mínimo e baixa escolaridade (menos de 8 anos). Essas condições em si já prenunciam riscos para diversos agravos à saúde. Quando se observa a cor da pele autodeclarada, mais de $80 \%$ dessas mulheres referem cor da pele preta, corroborando a ancestralidade africana, outro aspecto que, infelizmente, também já foi apontado como risco para agravantes na área da saúde.
Esse risco não se encontra associado a fatores biológicos, mas deve-se ao racismo institucional ${ }^{14}$. Um estudo de base populacional realizado com mulheres de 20 a 60 anos, no sul do Brasil, revelou que a probabilidade de as mulheres não realizarem os exames citopatológicos e de mamas foi significantemente maior nas negras, mesmo após ajustamento de variáveis sociais e econômicas. Para os autores, o diferencial observado pode ser um reflexo das desigualdades raciais e socioeconômicas vividas por mulheres negras no acesso aos serviços e ações de atenção à saúde reprodutiva ${ }^{3}$.

Nos resultados do presente estudo, as características das mulheres em relação à saúde reprodutiva apontam para um acesso restrito às atividades educativas ou de planejamento familiar. Mais da metade das mulheres tiveram sua primeira gestação ainda na adolescência e referem três ou mais gestações. Outro aspecto que define uma situação crítica é que o exame preventivo para o câncer do colo uterino não foi realizado por mais de $15 \%$ das mulheres e muitas o realizam de forma irregular. Esses resultados apontam para uma situação de negligência que é corroborada pelo relato de que mais de $60 \%$ das mulheres informaram que nunca tiveram suas mamas examinadas por profissionais de saúde. São dados que, efetivamente, reforçam as percepções de outros pesquisadores sobre desigualdades sociais e raciais vivenciadas por mulheres negras ${ }^{3,15}$.

É digno de análise mais criteriosa o fato de que mais de um quarto das mulheres já vivenciam situação de esterilidade, com registro de laqueadura tubária e/ou histerectomia. Não foi objetivo deste estudo avaliar as indicações dos referidos procedimentos, mas é preciso destacar que os números denotam uma interferência precoce no direito de procriação. Muitas mulheres costumam vivenciar sentimentos de arrependimento após procedimentos de esterilização definitiva como laqueadura tubária ${ }^{16}$.

Para avaliação específica do ciclo gravídico-puerperal, o presente trabalho limitou-se às mulheres com filhos menores de cinco anos, buscando minimizar limitações de memória. Embora esse fato tenha restringido o número de mães avaliadas, ainda foi possível registrar situações críticas de acesso e atenção à saúde da mulher e da criança. Mais de $20 \%$ das mulheres com crianças menores de cinco anos relataram menos de seis consultas pré-natais. Esses valores, associados ao registro de início tardio da assistência pré-natal para um terço das mulheres quilombolas, corroboram a existência de entraves no acesso e a indisponibilidade de serviços para a população avaliada. Situação similar já foi referida para outras comunidades de mulheres negras no país $3,17,18$.

É relevante destacar ainda a falta de acesso aos cuidados puerperais, pois quase metade das entrevistadas não passou por 
consultas puerperais, ocasião em que essas mulheres poderiam ser orientadas sobre diversos aspectos da sua saúde reprodutiva. Infelizmente, a situação verificada não é singular ou pontual. Outros estudos já apontaram que a as atividades de atenção à saúde da mulher são mais deficitárias em áreas rurais e particularmente mais críticas em comunidades mais pobres ${ }^{19,20}$.

O percentual elevado de crianças que nasceram de baixo peso (maior que a média nacional) é outro indicador de cuidados precários em relação ao ciclo gravídico-puerperal. O peso de nascimento é uma medida-resumo dos cuidados recebidos pela mãe durante a gestação e da saúde do concepto. Em um estudo conduzido em São Paulo, maiores taxas de nascimento de recém-nascidos de baixo peso e prematuros se mostraram estatisticamente associadas à cor da pele da mãe (preta e parda). Essa associação permaneceu mesmo após ajustamento dos dados em relação à renda familiar e escolaridade materna, o que sugere, segundo os autores, que iniquidades raciais e discriminação podem estar definindo esses resultados ${ }^{2}$.

Outros aspectos relacionados aos determinantes de saúde para a criança revelaram que, além da vulnerabilidade social e econômica, as crianças quilombolas residem em casas com elevada densidade populacional e mais de um quinto delas não contam com a presença do pai na mesma residência. Especificamente em relação aos cuidados de saúde, apesar da situação favorável em relação ao estado vacinal, elevado percentual de crianças não recebiam regularmente a suplementação de vitamina $\mathrm{A}$, conforme recomendação do Ministério da Saúde para a região ${ }^{21}$.

Além das questões de preconceito racial e cultural enfrentadas pela comunidade quilombola, a situação geográfica, de localização em meio rural, representa outro importante obstáculo para o acesso pleno aos cuidados de saúde. Beheregaray e Gerhardt ${ }^{22}$, avaliando especificamente a atenção materno-infantil em um município do Sul do Brasil, constataram que existe significativa condição de desvantagem para as comunidades rurais. Para as autoras, os serviços de saúde e os modelos assistenciais de atenção à saúde ainda são muito voltados para comunidades urbanas, fato que compromete, sobretudo, a integralidade da assistência. Deve-se considerar também que as particularidades culturais podem interferir na busca pelos serviços de saúde e que comunidades quilombolas apresentam menores taxas de utilização de serviços de saúde ${ }^{8}$.

Em outro estudo que investigou a percepção de mães quilombolas sobre os cuidados de saúde, os atributos da atenção primária foram avaliados por meio dos escores aferidos segundo o PCA-Tool (Primary Care Assessment Tool - Instrumento de Avaliação da Atenção Primária), validado internacionalmente e recomendado pelo Ministério da Saúde do Brasil. Verificou-se baixa fidelidade aos atributos da atenção primária, com piores escores para os atributos de Orientação Familiar e Acesso-acessibilidade. Para os autores, esses resultados também destacam a necessidade de maiores esforços para adequação do novo modelo assistencial à população estudada ${ }^{23}$.

É relevante destacar alguns aspectos positivos, como o fato de que $100 \%$ das crianças passaram por acompanhamento de puericultura, que quase todas as crianças se encontravam com vacinação em dia e que mais de $90 \%$ das famílias avaliadas contavam com benefício social do governo. Esses dados indicam que algumas medidas têm sido tomadas, sendo desejável que se incrementem para assegurar maior qualidade de vida às comunidades quilombolas.

Em síntese, os resultados observados registram um conjunto de carências e fragilidades que ainda apontam para um histórico de abandono das comunidades quilombolas, pelo menos em relação aos cuidados de saúde materno-infantil. A Política Nacional de Saúde Integral da População $\mathrm{Negra}^{11}$, que propõe ações transversais, no Sistema Único de Saúde (SUS), visando garantir a efetivação do direito à saúde da população negra em relação à promoção, à prevenção e ao tratamento dos agravos de saúde, parece ainda não ter se materializado. Enquanto o estímulo à criação de equipes específicas para as comunidades quilombolas se traduzir em simples repasse financeiro, sem acompanhamento e monitoramento mais estritos, as repercussões sobre o estado de saúde dessa população tendem a ser muito limitadas. Assim, se perpetuam as dificuldades de acesso, a falta de vínculos e responsabilização por parte dos profissionais de saúde. As questões apontadas são graves e ensejam a inserção de aspectos éticos na discussão das políticas específicas às comunidades quilombolas, buscando-se, inclusive, dar voz aos sujeitos envolvidos?.

Parece necessário, portanto, repensar a atenção à saúde para as comunidades quilombolas, considerando as suas particularidades, o contexto de localização predominantemente rural, as peculiaridades culturais, de acesso aos bens de consumo duráveis e serviços públicos, de oportunidades sociais, além das características epidemiológicas. Processos inclusivos e estratégias mais efetivas de promoção da equidade são imperativos para minimizar os danos recorrentes que o racismo institucional tem produzido às comunidades quilombolas. 


\section{REFERÊNCIAS}

1. Almeida WS, Szwarcwald CL. Mortalidade infantil e acesso geográfico ao parto nos municípios brasileiros. Rev Saúde Pública. 2012;46(1):68-76.

2. Silva LM, Silva RA, Silva AA, Bettiol H, Barbieri MA. Racial inequalities and perinatal health in the southeast region of Brazil. Braz J Med Biol Res. 2007;40(9):1187-94.

3. Bairros FS, Meneghel SN, Dias-da-Costa JS, Bassani DG, Menezes AMB, Gigante DP, et al. Racial inequalities in access to women's health care in southern Brazil. Cad Saúde Pública. 2011;27(12):2364-72.

4. Bastos JL, Celeste RK, Faerstein E, Barros AJ. Racial discrimination and health: a systematic review of scales with a focus on their psychometric properties. Soc Sci Med. 2010;70(7):1091-9.

5. Barata RB, Almeida MF, Montero CV, Silva ZP. Health inequalities based on ethnicity in individuals aged 15 to 64, Brazil, 1998. Cad Saúde Pública. 2007;23(2):305-13.

6. Batista LE, Escuder MML, Pereira JCR. A cor da morte: causas de óbito segundo características de raça no Estado de São Paulo, 1999 a 2001. Rev Saúde Pública. 2004;38(5):630-6.

7. Volochko A, Vidal NP. Desigualdades raciais na saúde: mortalidade nas regiões de saúde paulistas, 2005. BIS: Boletim do Instituto de Saúde. 2010;12(2):143-53

8. Gomes KO, Reis EA, Guimarães MDC, Cherchiglia ML. Utilização de serviços de saúde por população quilombola do Sudoeste da Bahia, Brasil. Cad Saúde Pública. 2013;29(9):1829-42.

9. Vieira ABD, Monteiro PS. Comunidade quilombola: análise do problema persistente do acesso à saúde, sob o enfoque da Bioética de Intervenção. Saúde debate. 2013;37(99):610-8.

10. Leite IB. Os quilombos no Brasil: questões conceituais e normativas. Etnográfica. 2000;4(2):333-54.

11. Brasil. Ministério da Saúde. Política Nacional de Saúde Integral da População Negra: uma política do SUS. Brasília: Ministério da Saúde; 2010

12. Cabral-Miranda G, Dattoli VCC, Dias-Lima A. Enteroparasitos e condições socioeconômicas e sanitárias em uma comunidade quilombola do semiárido baiano. Rev Patol Tropical. 2010;39(1):48-55.

13. Silva JAN. Condições Sanitárias e de saúde em Caiana dos Crioulos, uma comunidade quilombola do Estado da Paraíba. Saúde Soc. 2007;16(2):111-24.
14. López LC. The concept of institutional racism: applications within the healthcare field. Interface Comun Saúde Educ. 2012;16(40):121-34.

15. Góes EF, Nascimento ER. Mulheres negras e brancas e os níveis de acesso aos serviços preventivos de saúde: uma análise sobre as desigualdades. Saúde debate. 2013;37(99):571-9.

16. Ludermir AB, Machado KM, Costa AM, Alves SV, Araújo TV. Tubal ligation regret and related risk factors: findings from a casecontrol study in Pernambuco State, Brazil. Cad Saúde Pública. 2009;25(6):1361-8

17. Domingues RMSM, Leal MC, Hartz ZMA, Dias MAB, Vettore MV. Acesso e utilização de serviços de pré-natal na rede SUS do município do Rio de Janeiro, Brasil. Rev Bras Epidemiol. 2013; 16(4):953-65.

18. Martinelli KG, Santos Neto ET, Gama SGN, Oliveira AE. Adequação do processo da assistência pré-natal segundo os critérios do Programa de Humanização do Pré-natal e Nascimento e Rede Cegonha. Rev Bras Ginecol Obstet. 2014;36(2):56-64.

19. Cardoso LSM, Mendes LL, Velasquez-Melendez G. Diferenças na atenção pré-natal nas áreas urbanas e rurais do Brasil: estudo transversal de base populacional. Rev Min Enferm. 2013;17(1):93-100.

20. Mano PS, Cesar JA, González-Chica DA, Neumann NA. Iniquidade na assistência à gestação e ao parto em município do semiárido brasileiro. Rev Bras Saúde Mater Infant. 2011;11(4):381-8.

21. Brasil. Ministério da Saúde. Secretaria de Atenção à Saúde. Departamento de Atenção Básica. Vitamina A Mais: Programa Nacional de Suplementação de Vitamina A: Condutas Gerais. Brasília: Ministério da Saúde; 2004

22. Beheregaray LR, Gerhardt TE. A integralidade no cuidado à saúde materno-infantil em um contexto rural: um relato de experiência. Saúde Soc. 2010;19(1):201-12.

23. Marques AS, Freitas DA, Leão CDA, Oliveira SKM, Pereira MM, Caldeira AP. Atenção Primária e saúde materno-infantil: a percepção de cuidadores em uma comunidade rural quilombola. Ciên Saúde Coletiva. 2014;19(2):365-71.

Recebido em: 02/07/2014 Aprovado em: 01/09/2014 